\title{
Bioactive $p$-Terphenyl Derivatives from a Cordyceps-Colonizing Isolate of Gliocladium sp.
}

Huijuan Guo, ${ }^{\dagger, \dagger}$ Hejiao Hu, ${ }^{\dagger}$ Shuchun Liu, ${ }^{\dagger}$ Xingzhong Liu, ${ }^{\dagger}$ Yuguang Zhou, ${ }^{\dagger}$ and Yongsheng Che ${ }^{*, \dagger}$

Key Laboratory of Systematic Mycology and Lichenology, and Center for Bio-Energy and Industrial Microbiology, Institute of Microbiology, Chinese Academy of Sciences,

Beijing, 100080, People's Republic of China, and Graduate School of Chinese Academy of Sciences, Beijing, 100039, People's Republic of China

Contents

Page

1) Figure S1. ${ }^{1} \mathrm{H}$ NMR spectrum of gliocladinin $\mathrm{A}\left(\mathbf{1} ; 400 \mathrm{MHz}, \mathrm{CDCl}_{3}\right)$

2) Figure S2. ${ }^{13} \mathrm{C}$ NMR spectrum of gliocladinin $\mathrm{A}\left(\mathbf{1} ; 100 \mathrm{MHz}, \mathrm{CDCl}_{3}\right)$

3) Figure S3. CD spectrum of gliocladinin A $(\mathbf{1} ; \mathrm{MeOH})$

4) Figure S4. ${ }^{1} \mathrm{H}$ NMR spectrum of gliocladinin B $\left(2 ; 300 \mathrm{MHz}\right.$, Acetone- $\left.d_{6}\right)$

5) Figure S5. ${ }^{13} \mathrm{C}$ NMR spectrum of gliocladinin B (2; 75.5 MHz, Acetone- $\left.d_{6}\right) \quad 6$

\footnotetext{
* To whom correspondence should be addressed: Phone: 861082618785.

Fax: 8610 82618785. E-mail: cheys@im.ac.cn.

${ }^{\dagger}$ Institute of Microbiology, Chinese Academy of Sciences.

${ }^{\ddagger}$ Graduate School of Chinese Academy of Sciences.
} 


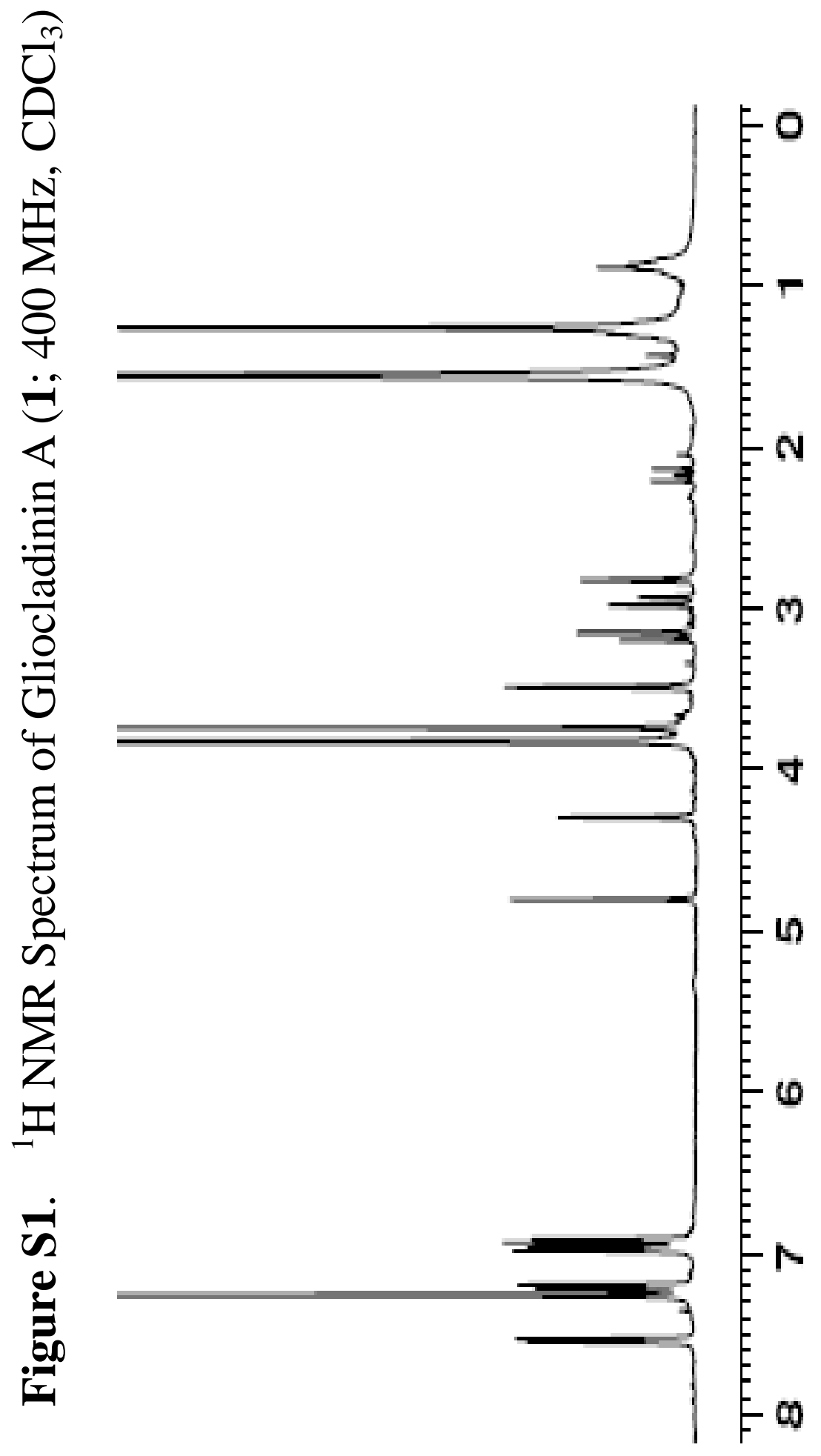



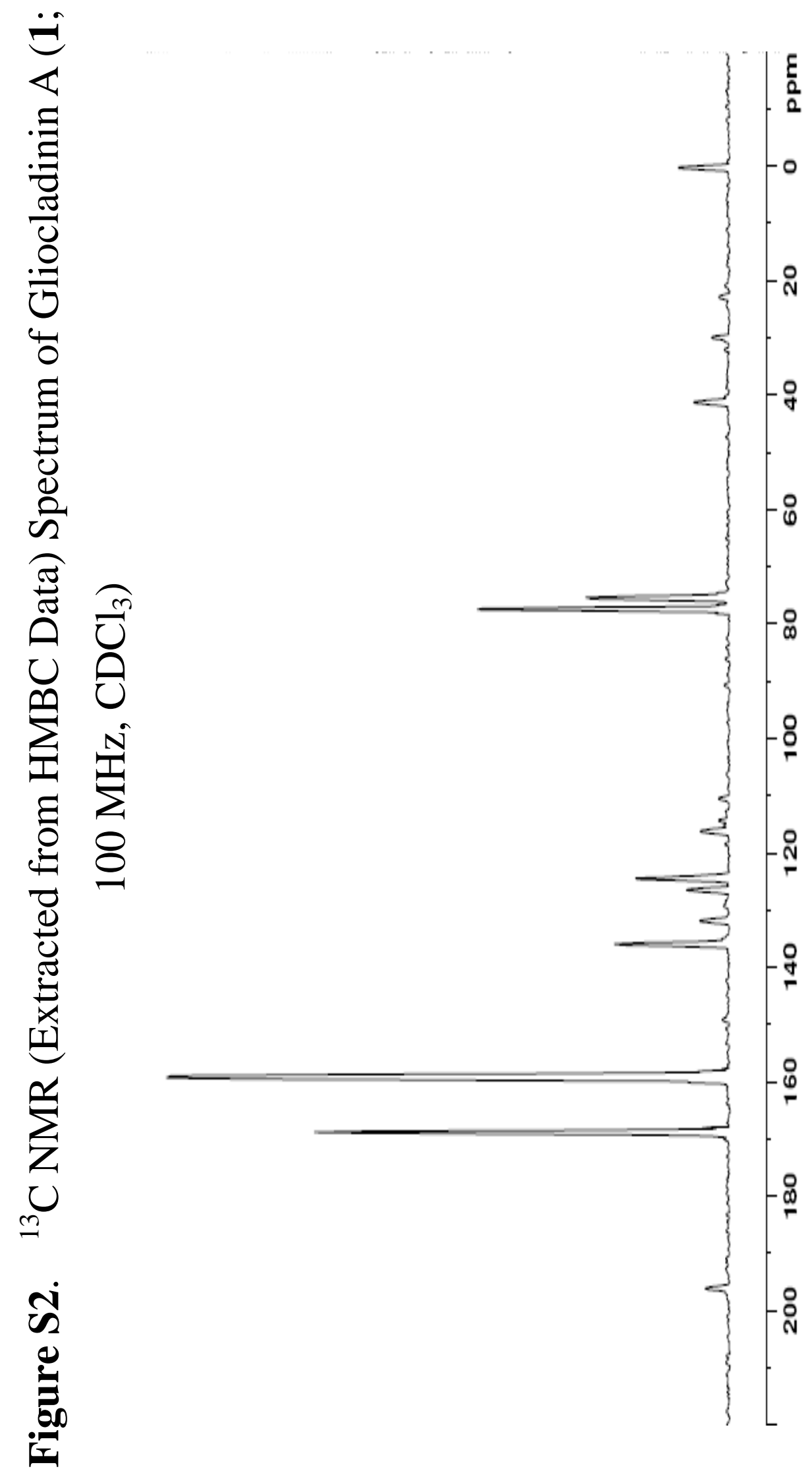

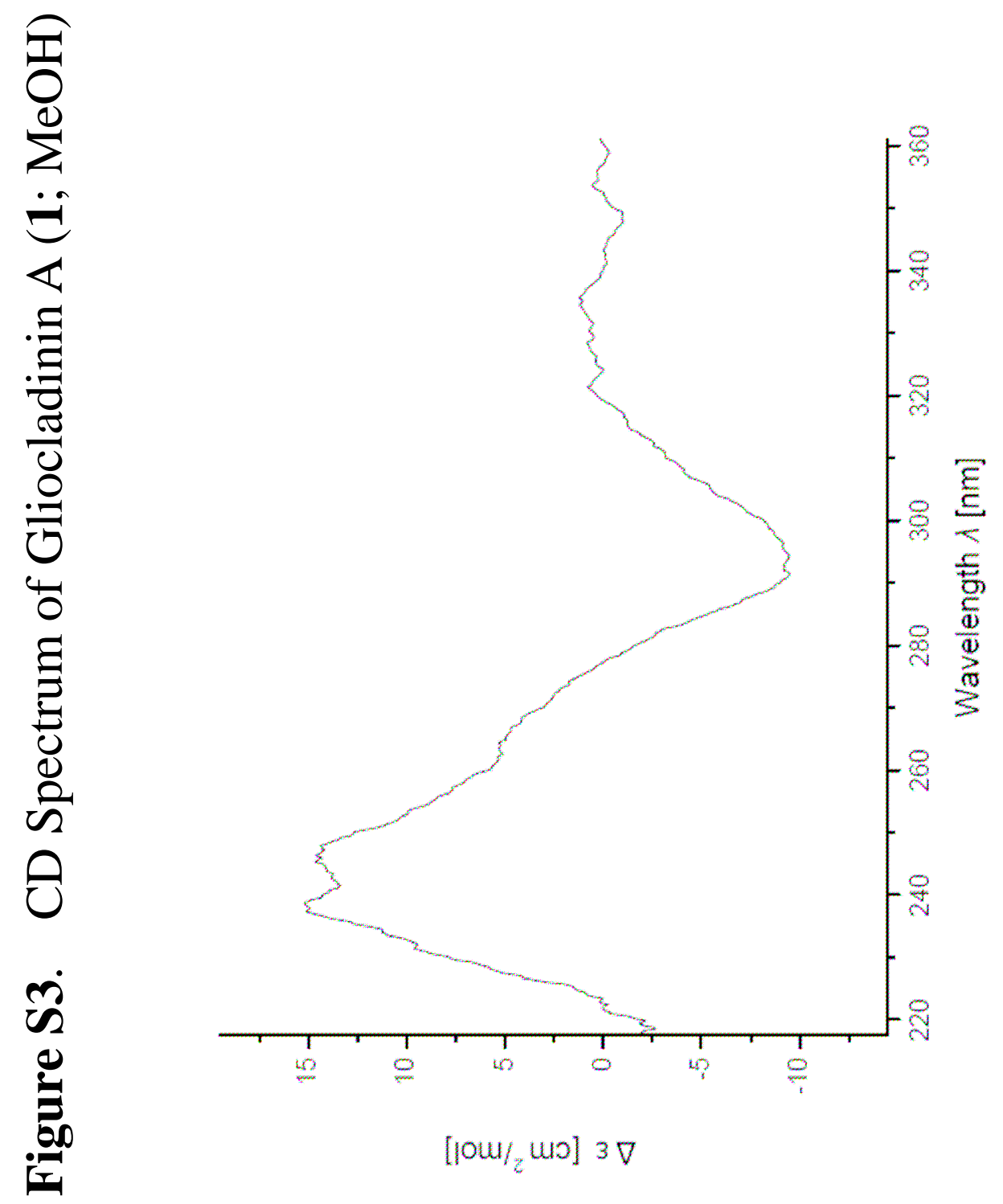


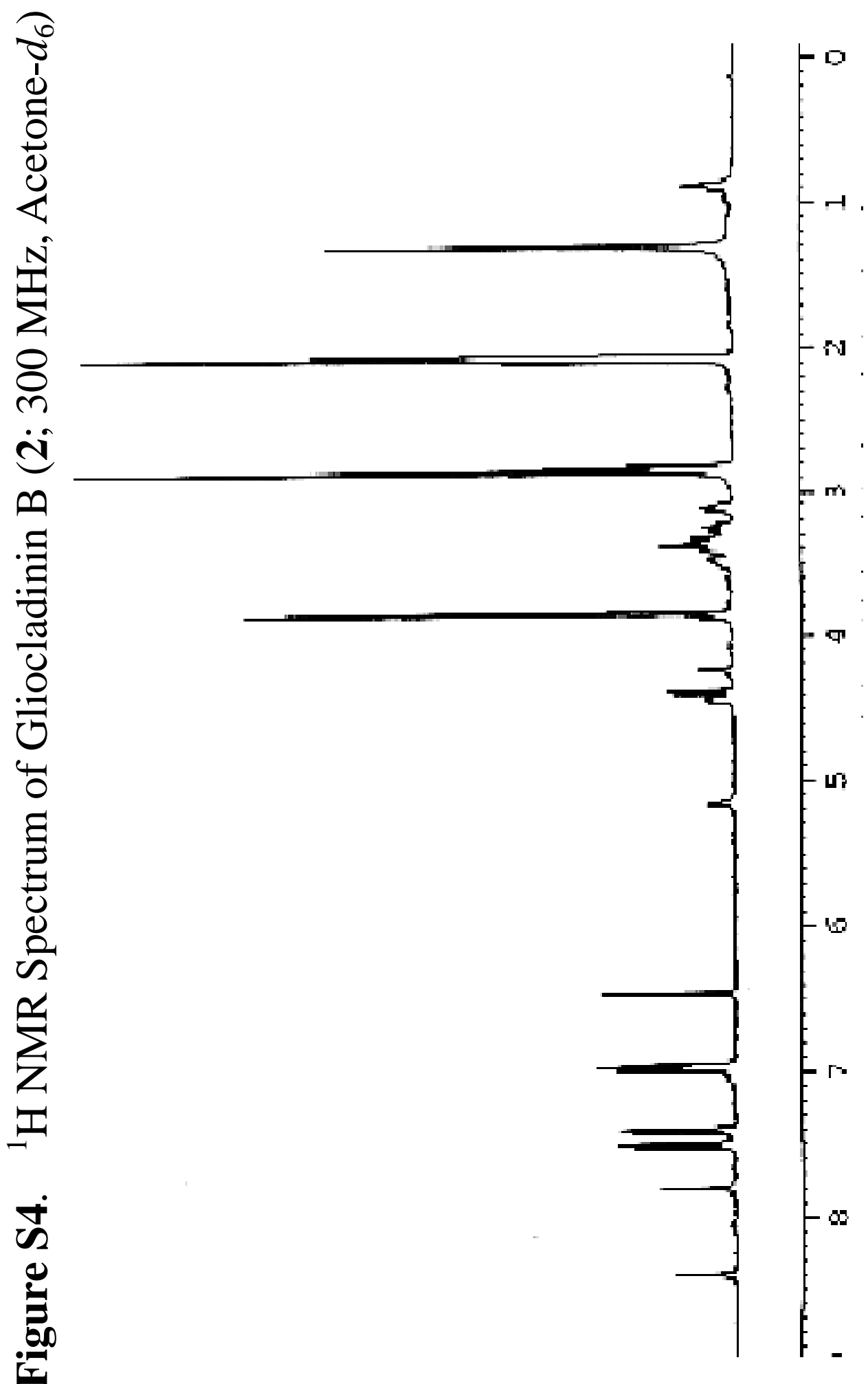




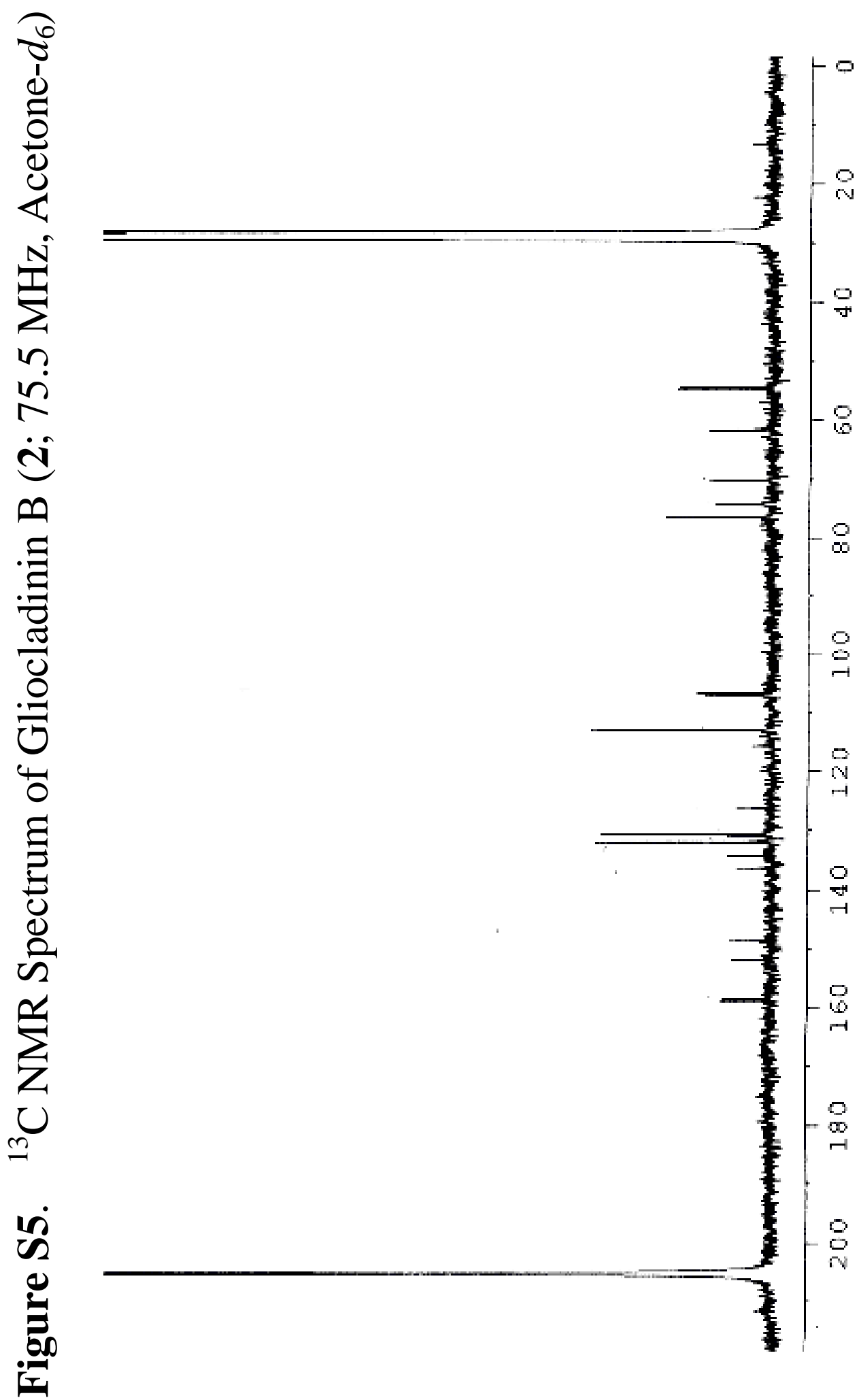

